\title{
A Cooperative Diversity Analysis of Two User Mobile Communication System with Maximal Ratio Combining
}

\author{
Sateeshkrishna Dhuli, V. V. Mani \\ Department of Electronics and Communication Engineering, National Institute of Technology, Warangal, India \\ Email: dvskrishna.nitw@gmail.com,vvmani@nitw.ac.in
}

Received August 11, 2012; revised September 11, 2012; accepted October 11, 2012

Copyright (C) 2013 Sateeshkrishna Dhuli, V. V. Mani. This is an open access article distributed under the Creative Commons Attribution License, which permits unrestricted use, distribution, and reproduction in any medium, provided the original work is properly cited.

\begin{abstract}
Cooperative communication is going to play a vital role in the next generation wireless networks. In this paper we derive the expression for symbol error probability (SEP) of a two-user cooperative diversity system, where two users cooperate through the decode-and-forward (DF) relaying with binary phase-shift keying (BPSK) modulation in a flat Rayleigh fading environment. We compare the computational results obtained by the SEP expression with the simulation results using maximal-ratio combining (MRC), equal-gain combining (EGC) and selection combining (SC) techniques. Numerical results show the performance of a cooperative diversity system with maximal-ratio combining is giving better results compared to SC and EGC techniques.
\end{abstract}

Keywords: Symbol Error Probability (SEP); Maximal-Ratio Combining (MRC); Equal-Gain Combining (EGC); Selection Combining (SC); Decode-and-Forward (DF) Relaying

\section{Introduction}

Cooperative diversity is a new form of spatial diversity where diversity is achieved through the cooperation between users presented in the network. The key idea behind this technology is sharing the power, computation and antenna resources of the neighboring users in the network. It is also going to be a promising alternative to combat the multipath fading and to provide the reliable communication [1]. An analytical study about the user cooperation is first discussed in [2]. The amplify-andforward (AF), decode-and-forward (DF) and coded cooperation methods are discussed in [3]. In [4], SEP is derived for a two-user cooperative diversity system. In [5], some new closed form expressions are derived in a flat Rayleigh fading environment. In this paper we consider a fundamental cooperative diversity system, where two users cooperating through the DF relaying with BPSK modulation in a flat Rayleigh fading environment. The rest of the paper has been organized as follows. In Section 2, system model of a fundamental cooperative diversity system is discussed and mathematical expressions are given. In Section 3 different combining techniques are discussed. In Section 4 we derived the SEP expression for a two-user cooperative diversity system. Simulation results are presented in Section 5. In Section 6 we have given the conclusions.

\section{System Model}

We consider a cooperative diversity system with two users and a single destination. Let us assume user 1 acts as a source and user 2 relays the data received from user 1 to the destination. In time frame 1 , user 1 transmits the data $x_{1}$ to the destination directly as well as to the user 2. In time frame 2, user 2 decodes the data $x_{1}$ and forwards as $\hat{x}_{1}$ to the destination as shown in the Figure 1.

$$
\begin{aligned}
& r_{1 d}=h_{1 d} X_{1}+n_{1 d} \\
& r_{12}=h_{12} X_{1}+n_{12} \\
& r_{2 d}=h_{2 d} \hat{x}_{1}+n_{2 d}
\end{aligned}
$$

$r_{1 d}, r_{12}$ are the received complex baseband signals at the destination and user 2 respectively in time frame $1 . r_{2 d}$ is the complex baseband signal at the destination in time frame 2. $h_{1 d}, h_{12}$ are complex fading gains from user 1 to destination and from user 1 to user 2 respectively. $h_{2 d}$ is the complex fading gain from user 2 to destination. $x_{1}$ is the transmitted BPSK symbol of user 1 having energy $2 E_{s}\left(x_{1} \in\left\{-\sqrt{2 E_{s}}, \sqrt{2 E_{s}}\right\}\right), n_{1 d}$ and $n_{12}$ are the addi- 


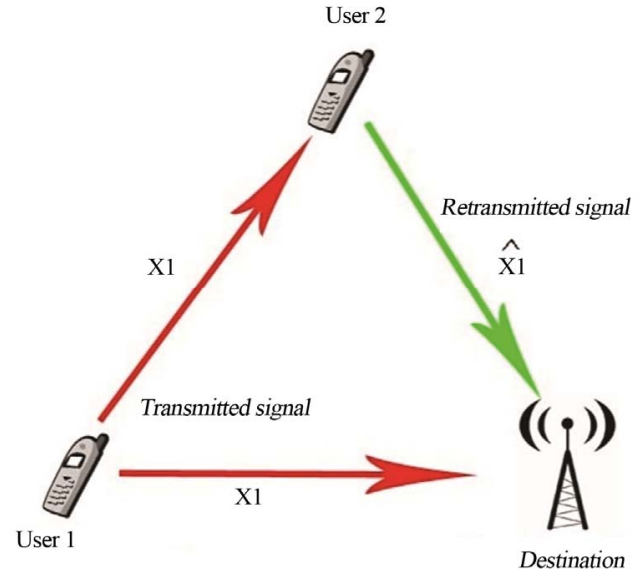

Figure 1. Two-user cooperative diversity system.

tive white Gaussian noises from user 1 to destination and from user 1 to user 2 respectively. $n_{2 d}$ is the additive white Gaussian noise from user 2 to destination. $h_{1 d}, h_{12}, h_{2 d}$ are the independent zero-mean complex circular Gaussian random variables having variances.

$\Omega_{1 d}, \Omega_{12}, \Omega_{2 d}$ respectively and are independent of the additive noises. $n_{1 d}, n_{12}, n_{2 d}$ are independent and identically distributed zero-mean complex circular Gaussian random variables with variance $2 N_{0}$, i.e. having a $C \mathcal{N}\left(0,2 N_{0}\right)$ distribution.

\section{Combining Techniques}

\subsection{Selection Combining}

In the cooperation mode the data is sent by the user 1 is decoded as $\hat{x}_{1}$ at user 2, which can be expressed as

$$
\hat{x}_{1}=\sqrt{2 E_{s}} \operatorname{sgn}\left(\operatorname{Re}\left(h_{12}^{*} r_{12}\right)\right)
$$

In the non cooperation mode, the data received directly from user 1 at the destination. The decoded symbol obtained by the coherent detection is $\hat{\hat{x}}_{1}$, which can be expressed as

$$
\hat{\hat{x}}_{1}=\sqrt{2 E_{s}} \operatorname{sgn}\left(\operatorname{Re}\left(h_{1 d}^{*} r_{1 d}\right)\right)
$$

$\operatorname{sgn}(\cdot)$ denotes the signum function. Let $\hat{\hat{x}}_{s c}$ denotes the final decoded symbol at the destination using $\mathrm{SC}$ is given by

$$
\hat{\hat{x}}_{s c}=\left\{\begin{array}{l}
\sqrt{2 E_{s}} \operatorname{sgn}\left(\operatorname{Re}\left(h_{1 d}^{*} r_{1 d}\right)\right),\left|h_{1 d}\right|^{2}>\left|h_{2 d}\right|^{2} \\
\sqrt{2 E_{s}} \operatorname{sgn}\left(\operatorname{Re}\left(h_{2 d}^{*} r_{2 d}\right)\right),\left|h_{1 d}\right|^{2}<\left|h_{2 d}\right|^{2}
\end{array}\right.
$$

\subsection{Equal-Gain Combining}

In this technique signals received at the destination are multiplied by a complex weighting factor that compensates the phase rotation of the channel. Let $\hat{\hat{x}}_{\text {egc }}$ denotes the output of the EGC, which can be expressed as

$$
\hat{\hat{x}}_{\text {egc }}=\sqrt{2 E_{s}} \operatorname{sgn}\left(\operatorname{Re}\left(\sum_{l=1}^{2} \alpha_{l} r_{l d}\right)\right)
$$

$\alpha_{l}$ is the weighting factor of EGC, which can be given by

$$
\alpha_{l}=\mathrm{e}^{-j \phi_{l d}}
$$

where phase $\phi_{l d}=\angle h_{l d}$ for $l=1,2 .\left|\alpha_{1}\right|,\left|\alpha_{2}\right|$ are the magnitudes of the weighting factors which are same and do not depend on the signal-to-noise ratio (SNR) values of the communication links.

\subsection{Maximal-Ratio Combining}

Let $\hat{\hat{x}}_{m r c}$ denotes final decoded symbol at the destination using MRC is given by

$$
\hat{\hat{x}}_{m r c}=\sqrt{2 E_{s}} \operatorname{sgn}\left(\operatorname{Re}\left(\sum_{k=1}^{2} \alpha_{k} r_{k d}\right)\right)
$$

$\alpha_{k}$ is the weighting factor of MRC, which can be expressed as

$$
\alpha_{k}=\frac{h_{k d}^{*}}{\Omega_{k d}}=\frac{\left|h_{k d}\right| \mathrm{e}^{-j \phi_{k d}}}{\Omega_{k d}}
$$

where $\Omega_{k d}$ is the variance of $h_{k d}$, phase $\phi_{k d}=\angle h_{k d}$ for $k=1,2$.

\section{Error Analysis}

\subsection{Non-Cooperation Mode}

The SEP conditioned on $h_{1 d}$, obtained by the coherent detection is given by

$$
P_{e 1 d}\left(h_{1 d}\right)=Q\left(\sqrt{\frac{2 E_{s}\left|h_{1 d}\right|^{2}}{N_{0}}}\right)
$$

where $Q(\cdot)$ denotes the Gaussian $Q$-function.

The instantaneous SNR of the user 1 to destination link is denoted as

$$
\gamma_{1 d}=\frac{E_{s}\left|h_{1 d}\right|^{2}}{N_{0}}
$$

The average SNR of the user 1 to destination link is denoted as

$$
\Gamma_{1 d}=\frac{E_{s} \Omega_{1 d}}{N_{0}}
$$

Therefore (12) can be written as

$$
P_{e 1 d}\left(\gamma_{1 d}\right)=Q\left(\sqrt{2 \gamma_{1 d}}\right)
$$

using Craig's formula (14) can be written as

$$
P_{e l d}\left(\gamma_{1 d}\right)=\frac{1}{\pi} \int_{0}^{\frac{\pi}{2}} \exp \left(-\frac{\gamma_{1 d}}{\sin ^{2} \theta}\right) \mathrm{d} \theta
$$


After averaging the (15) over the statistics of $\gamma_{1 d}$, we obtain SEP in the non-cooperation mode as

$$
P_{\text {eld }}=\frac{1}{2}\left(1-\sqrt{\frac{\Gamma_{1 d}}{1+\Gamma_{1 d}}}\right)
$$

\subsection{Cooperation Mode}

Let $\gamma_{12}$ and $\gamma_{2 d}$ denote the instantaneous SNR of the user 1 to user 2 link and instantaneous SNR of the user 2 to destination link respectively, given by

$$
\gamma_{12}=\frac{E_{s}\left|h_{1 d}\right|^{2}}{N_{0}}, \gamma_{2 d}=\frac{E_{s}\left|h_{2 d}\right|^{2}}{N_{0}}
$$

Let $\Gamma_{12}$ and $\Gamma_{2 d}$ denote the average SNR of the user 1 to user 2 link and average SNR of the user 2 to destination link respectively, given by

$$
\Gamma_{12}=\frac{E_{s} \Omega_{12}}{N_{0}}, \Gamma_{2 d}=\frac{E_{s} \Omega_{2 d}}{N_{0}}
$$

We consider the case when user 1 transmits symbol $\sqrt{2 E_{s}}$, the disjoint events which lead to a correct decision can be enumerated as

$$
\begin{gathered}
\varepsilon_{1}=\left\{\operatorname{Re}\left(h_{1 d}^{*} r_{1 d}\right)>0, \operatorname{Re}\left(h_{2 d}^{*} r_{2 d}\right)>0 \mid x_{1}=\sqrt{2 E_{s}}\right\} \\
\varepsilon_{2}=\left\{\operatorname{Re}\left(h_{1 d}^{*} r_{1 d}\right)>0, \operatorname{Re}\left(h_{2 d}^{*} r_{2 d}\right)<0, \gamma_{1 d}>\gamma_{2 d} \mid x_{1}=\sqrt{2 E_{s}}\right\} \\
\varepsilon_{3}=\left\{\operatorname{Re}\left(h_{1 d}^{*} r_{1 d}\right)<0, \operatorname{Re}\left(h_{2 d}^{*} r_{2 d}\right)>0, \gamma_{1 d}<\gamma_{2 d} \mid x_{1}=\sqrt{2 E_{s}}\right\}
\end{gathered}
$$

Probability of the event $\varepsilon_{1}$ conditioned on $\gamma_{1 d}, \gamma_{12}$ and $\gamma_{2 d}$ can be written as

$$
\begin{aligned}
\operatorname{Pr}\left(\varepsilon_{1}\right)= & \left(1-P_{e 1 d}\left(\gamma_{1 d}\right)\right)\left(\left(1-P_{e 2 d}\left(\gamma_{2 d}\right)\right)\left(1-P_{e 12}\left(\gamma_{12}\right)\right)\right) \\
& +P_{e 2 d}\left(\gamma_{2 d}\right) P_{e 12}\left(\gamma_{12}\right)
\end{aligned}
$$

$\rho_{1 d}, \rho_{2 d}$ and $\rho_{12}$ are defined as

$$
\rho_{1 d} \triangleq \sqrt{\frac{\Gamma_{1 d}}{1+\Gamma_{1 d}}}, \rho_{2 d} \triangleq \sqrt{\frac{\Gamma_{2 d}}{1+\Gamma_{2 d}}}, \rho_{12} \triangleq \sqrt{\frac{\Gamma_{12}}{1+\Gamma_{12}}}
$$

Averaging (22) over the exponential statistics of $\gamma_{1 d}, \gamma_{2 d}$ and $\gamma_{12}$, we obtain the probability of the event $\varepsilon_{1}$ as

$$
\operatorname{Pr}\left(\varepsilon_{1}\right)=\frac{1}{4}\left(1+\rho_{1 d}\right)\left(1+\rho_{2 d} \rho_{12}\right)
$$

Probability of the event $\varepsilon_{2}$ can be written as

$$
\begin{aligned}
\operatorname{Pr}\left(\varepsilon_{2}\right)= & \left(1-P_{e 1 d}\left(\gamma_{1 d}\right)\right)\left(P_{e 2 d}\left(\gamma_{2 d}\right)\left(1-P_{e 12}\left(\gamma_{12}\right)\right)\right. \\
& \left.+\left(1-P_{e 2 d}\left(\gamma_{2 d}\right)\right) P_{e 12}\left(\gamma_{12}\right)\right)
\end{aligned}
$$

Averaging (25) over the statistics of $\gamma_{1 d}, \gamma_{2 d}$ and $\gamma_{12}$ under the condition $\gamma_{1 d}>\gamma_{2 d}$ can be simplified into

$$
\begin{aligned}
\operatorname{Pr}\left(\varepsilon_{2}\right)= & \frac{1}{2} \int_{0}^{\infty}\left(1-\rho_{12}+2 \rho_{12} Q(\sqrt{2 v})\right) \times \frac{1}{\Gamma_{2 d}} \mathrm{e}^{-\frac{v}{\Gamma_{2 d}}} \\
& \times\left[\int_{u=v}^{\infty}(1-Q(\sqrt{2 u})) \times \frac{1}{\Gamma_{1 d}} \mathrm{e}^{-\frac{u}{\Gamma_{1 d}}} \mathrm{~d} u\right] \mathrm{d} v
\end{aligned}
$$

Using the integration by parts we get

$$
\operatorname{Pr}\left(\varepsilon_{2}\right)=(1-Q(\sqrt{2 v})) \mathrm{e}^{\frac{-v}{\Gamma_{1 d}}}+\rho_{1 d} Q\left(\frac{\sqrt{2 v}}{\rho_{1 d}}\right)
$$

After applying the formula [4], we get

$$
\begin{aligned}
\operatorname{Pr}\left(\varepsilon_{2}\right) & =\frac{\Gamma_{1 d}\left(1-\rho_{12}\right)}{2\left(\Gamma_{1 d}+\Gamma_{2 d}\right)}+\frac{\Gamma_{1 d}\left(3 \rho_{12}-1\right)}{\left(\Gamma_{1 d}+\Gamma_{2 d}\right)} I\left(\sqrt{\frac{\Gamma_{1 d} \Gamma_{2 d}}{\Gamma_{1 d}+\Gamma_{2 d}}}, 0\right) \\
& +\rho_{1 d}\left(1-\rho_{12}\right) I\left(\frac{\sqrt{\Gamma_{2 d}}}{\rho_{1 d}}, 0\right) \\
& -\frac{\Gamma_{1 d} \rho_{12}}{\left(\Gamma_{1 d}+\Gamma_{2 d}\right)} I\left(\sqrt{\frac{\Gamma_{1 d} \Gamma_{2 d}}{\Gamma_{1 d}+\Gamma_{2 d}}}, \sqrt{\frac{\Gamma_{1 d} \Gamma_{2 d}}{\Gamma_{1 d}+\Gamma_{2 d}}}\right) \\
& +\rho_{1 d} \rho_{12} I\left(\sqrt{\Gamma_{2 d}}, \frac{\sqrt{\Gamma_{2 d}}}{\rho_{1 d}}\right)
\end{aligned}
$$

where quantities $\rho_{12 d}$ and $\epsilon_{12 d}$ are defined as

$$
\rho_{12 d} \triangleq \sqrt{\frac{\Gamma_{12} \Gamma_{2 d}}{\Gamma_{1 d}+\Gamma_{2 d}+\Gamma_{1 d} \Gamma_{2 d}}}, \epsilon_{12 d} \triangleq \sqrt{\frac{\Gamma_{2 d}}{\Gamma_{1 d}+\Gamma_{2 d}}}
$$

We finally obtain the probability of event $\varepsilon_{2}$

$$
\begin{aligned}
\operatorname{Pr}\left(\varepsilon_{2}\right) & \frac{\left(1-\epsilon_{12 d}\right)\left(1-\rho_{12}\right)}{2}+\frac{\left(1-\epsilon_{12 d}\right)\left(1-\rho_{12 d}\right)\left(3 \rho_{12}-1\right)}{4} \\
& +\frac{\rho_{1 d}\left(1-\rho_{12}\right)}{4}\left(1-\frac{\rho_{2 d}}{\sqrt{\rho_{1 d}^{2}+\rho_{2 d}^{2}-\rho_{1 d}^{2} \rho_{2 d}^{2}}}\right) \\
& -\left(1-\epsilon_{12 d}\right) \rho_{12}\left(\frac{1}{4}-\frac{\rho_{12 d}}{\pi} \tan ^{-1}\left(\frac{1}{\rho_{12 d}}\right)\right) \\
& +\frac{\rho_{1 d} \rho_{12}}{4}-\frac{\rho_{1 d} \rho_{2 d} \rho_{12}}{2 \pi} \tan ^{-1}\left(\frac{\rho_{1 d}}{\rho_{2 d}}\right) \\
& -\frac{\rho_{1 d} \rho_{2 d} \rho_{12}}{2 \pi \sqrt{\rho_{1 d}^{2}+\rho_{2 d}^{2}-\rho_{1 d}^{2} \rho_{2 d}^{2}}} \times \tan ^{-1}\left(\frac{\sqrt{\rho_{1 d}^{2}+\rho_{2 d}^{2}-\rho_{1 d}^{2} \rho_{2 d}^{2}}}{\rho_{1 d} \rho_{2 d}}\right)
\end{aligned}
$$

Probability of event $\varepsilon_{3}$ can be similarly written as

$$
\begin{aligned}
\operatorname{Pr}\left(\varepsilon_{3}\right)= & P_{e 1 d}\left(\gamma_{1 d}\right)\left(\left(1-P_{e 2 d}\left(\gamma_{2 d}\right)\right)\left(1-P_{e 12}\left(\gamma_{12}\right)\right)\right. \\
& \left.+P_{e 2 d}\left(\gamma_{2 d}\right) P_{e 12}\left(\gamma_{12}\right)\right)
\end{aligned}
$$


Averaging the (31) over the statistics of $\gamma_{1 d}, \gamma_{2 d}$ and $\gamma_{12}$ under the condition $\gamma_{1 d}<\gamma_{2 d}$ can be simplified into

$$
\operatorname{Pr}\left(\varepsilon_{3}\right)=\frac{1}{2} \int_{u=0}^{\infty} Q(\sqrt{2 u}) \frac{1}{\Gamma_{1 d}} \mathrm{e}^{-\frac{u}{\Gamma_{1 d}}} \times\left[\int_{v=u}^{\infty}\left(1+\rho_{12}-2 \rho_{12} Q(\sqrt{2 v})\right) \times \frac{1}{\Gamma_{2 d}} \mathrm{e}^{-\frac{v}{\Gamma_{2 d}}} \mathrm{~d} v\right] \mathrm{d} u
$$

Using the integration by parts we get

$$
\begin{aligned}
& \operatorname{Pr}\left(\varepsilon_{3}\right) \\
& =\frac{\left(1+\rho_{12}\right) \epsilon_{12 d}\left(1-\rho_{12 d}\right)}{8}-\rho_{12} \epsilon_{12 d} I\left(\sqrt{\frac{\Gamma_{1 d} \Gamma_{2 d}}{\Gamma_{1 d}+\Gamma_{2 d}}}, \sqrt{\frac{\Gamma_{1 d} \Gamma_{2 d}}{\Gamma_{1 d}+\Gamma_{2 d}}}\right)-\rho_{12} \rho_{2 d} I\left(\sqrt{\Gamma_{1 d}}, \frac{\sqrt{\Gamma_{1 d}}}{\rho_{2 d}}\right)
\end{aligned}
$$

Applying the formula as in [4], we get the probability of $\varepsilon_{3}$

$$
\begin{aligned}
& \operatorname{Pr}\left(\varepsilon_{3}\right) \\
= & \frac{\epsilon_{12 d}\left(1-\rho_{12 d}\right)\left(1+\rho_{12}\right)}{4}-\epsilon_{12 d} \rho_{12}\left(\frac{1}{4}-\frac{\rho_{12 d}}{\pi} \tan ^{-1}\left(\frac{1}{\rho_{12 d}}\right)\right)+\frac{\rho_{2 d} \rho_{12}}{4}-\frac{\rho_{1 d} \rho_{2 d} \rho_{12}}{2 \pi} \tan ^{-1}\left(\frac{\rho_{2 d}}{\rho_{1 d}}\right) \\
& -\frac{\rho_{1 d} \rho_{2 d} \rho_{12}}{2 \pi \sqrt{\rho_{1 d}^{2}+\rho_{2 d}^{2}-\rho_{1 d}^{2} \rho_{2 d}^{2}}} \times \tan ^{-1}\left(\frac{\sqrt{\rho_{1 d}^{2}+\rho_{2 d}^{2}-\rho_{1 d}^{2} \rho_{2 d}^{2}}}{\rho_{1 d} \rho_{2 d}}\right)
\end{aligned}
$$

The probability of correct decision is given by $\operatorname{Pr}\left(\varepsilon_{1}\right)+\operatorname{Pr}\left(\varepsilon_{2}\right)+\operatorname{Pr}\left(\varepsilon_{3}\right)$. Therefore the end-to-end SEP, which is denoted as $P_{e}$ in cooperation mode can be expressed as

$$
P_{e}=1-\left(\operatorname{Pr}\left(\varepsilon_{1}\right)+\operatorname{Pr}\left(\varepsilon_{2}\right)+\operatorname{Pr}\left(\varepsilon_{3}\right)\right)
$$

After substituting the (24), (30) and (34) in (35) we finally obtain the end-to-end SEP expression as

$$
\begin{aligned}
& P_{e} \\
& =\frac{1}{2}-\frac{\rho_{1 d}}{2}-\frac{\rho_{2 d} \rho_{12}}{2}-\frac{\rho_{12 d}}{4}+\frac{\epsilon_{12 d} \rho_{12 d}\left(1-\rho_{12}\right)}{2}+\rho_{12 d} \rho_{12}\left(\frac{3}{4}-\frac{1}{\pi} \tan ^{-1}\left(\frac{1}{\rho_{12 d}}\right)\right) \\
& +\frac{\rho_{1 d} \rho_{2 d}}{\sqrt{\rho_{1 d}^{2}+\rho_{2 d}^{2}-\rho_{1 d}^{2} \rho_{2 d}^{2}}}\left[\frac{\left(1-\rho_{12}\right)}{4}+\frac{\rho_{12}}{\pi} \tan ^{-1}\left(\frac{\sqrt{\rho_{1 d}^{2}+\rho_{2 d}^{2}-\rho_{1 d}^{2} \rho_{2 d}^{2}}}{\rho_{1 d} \rho_{2 d}}\right)\right]
\end{aligned}
$$

For $\Gamma_{1 d}=\Gamma_{2 d} \gg 1, \Gamma_{12} \gg 1$ the expression (36) can be approximated as

$$
P_{e} \approx \frac{1}{16 \Gamma_{1 d}^{2}}+\frac{1}{8 \Gamma_{12}}
$$

\section{Simulation Results}

In this section we show the numerical results of the SEP vs average SNR for BPSK modulation scheme. From the Figure 2, we observe the performance of the cooperation mode is better than the non-cooperation mode over a large range of SNR values when the $\Gamma_{12}$ value increases. From the Figure 3, we observe that the MRC improves the diversity significantly over EGC and SC, when $\Gamma_{12}=15 \mathrm{~dB}$ and $\Gamma_{1 d}$ is varied between 5 to 15 $\mathrm{dB}$. We also observe similar increase in performance when $\Gamma_{12}=25 \mathrm{~dB}$ and $\Gamma_{1 d}$ is varied between 5 to 20
dB. From the Figure 4, we also observe that for every 5 $\mathrm{dB}$ increase of $\Gamma_{12}$ The diversity range offered by MRC is approximately increases by $5 \mathrm{~dB}$. SEP values of EGC are almost close to the MRC because both the schemes are coherently combining the signals at the destination. The performance of the MRC, EGC, SC is almost similar after the points of intersection

$\left(\Gamma_{1 d}, \Gamma_{12}\right)=(15 \mathrm{~dB}, 15 \mathrm{~dB})$ and

$\left(\Gamma_{1 d}, \Gamma_{12}\right)=(20 \mathrm{~dB}, 25 \mathrm{~dB})$.

\section{Conclusions}

We investigated the performance of a two-user cooperative diversity system using MRC, EGC, SC techniques. First we compared the cooperation and non-cooperation modes for different $\Gamma_{12}$ values. We also compared the MRC, EGC, SC with each other in providing better diversity. The obtained simulation and computation results 


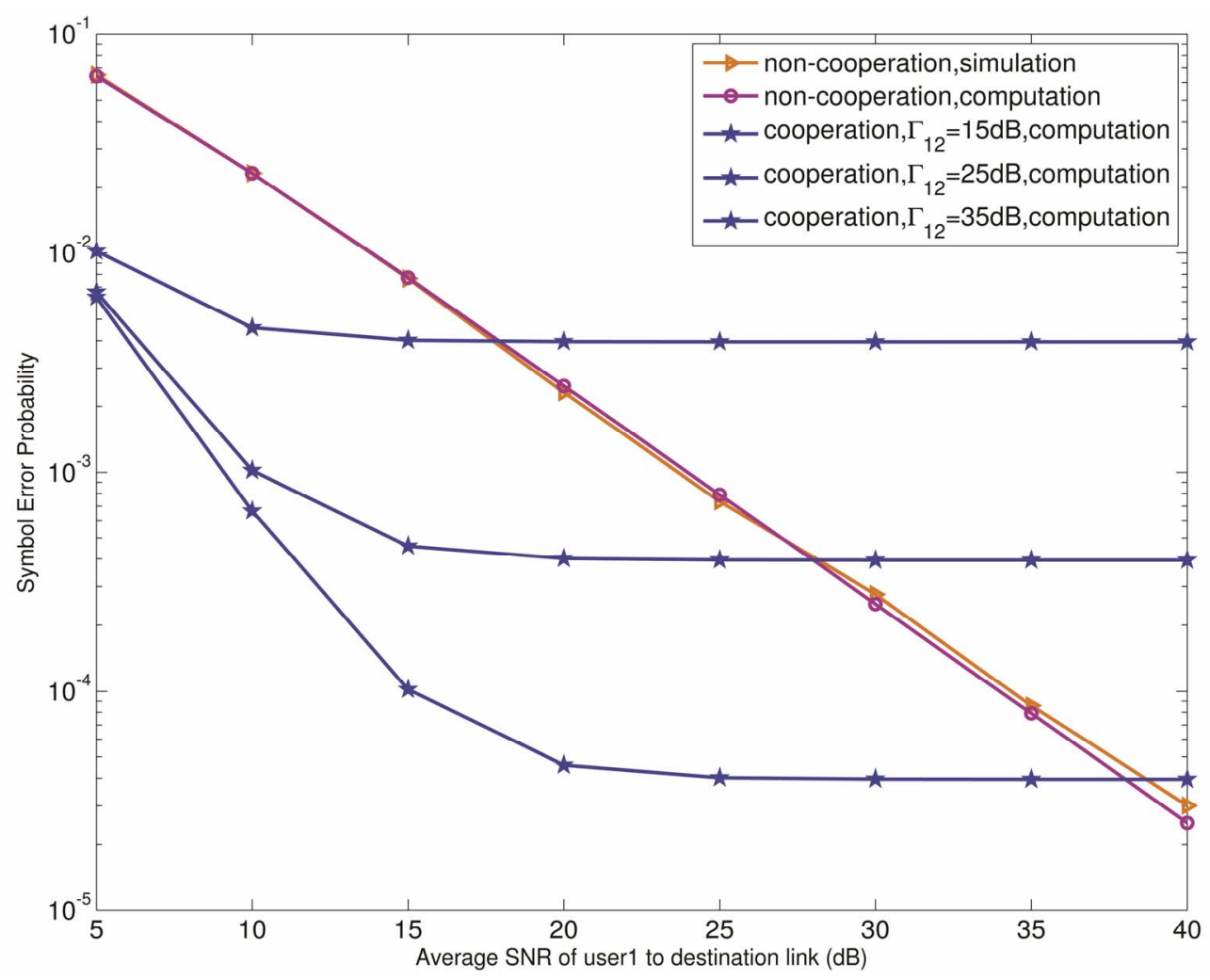

Figure 2. Comparison of cooperation and non-cooperation modes.

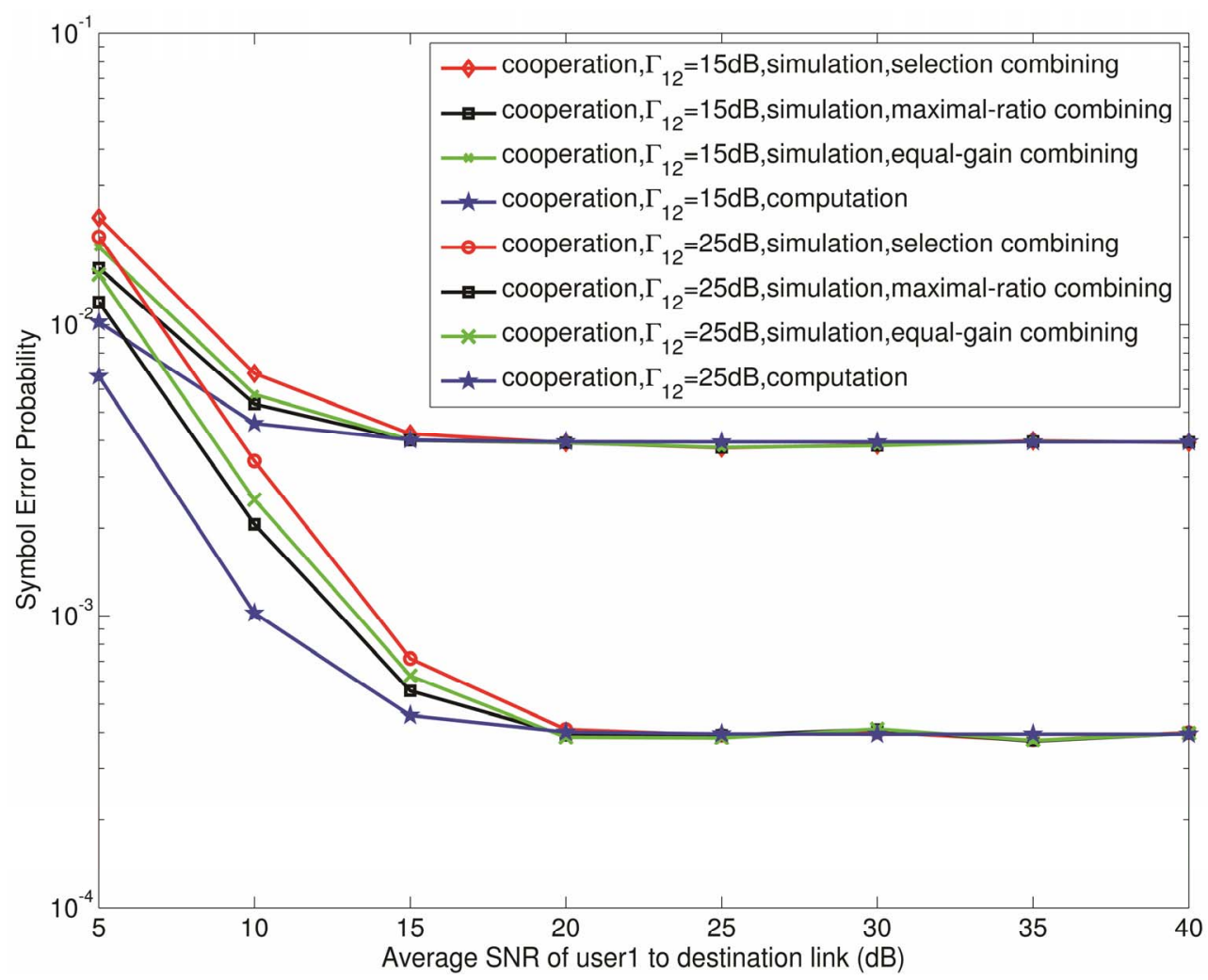

Figure 3. Comparison of computation and simulation results with MRC, EGC and SC. 


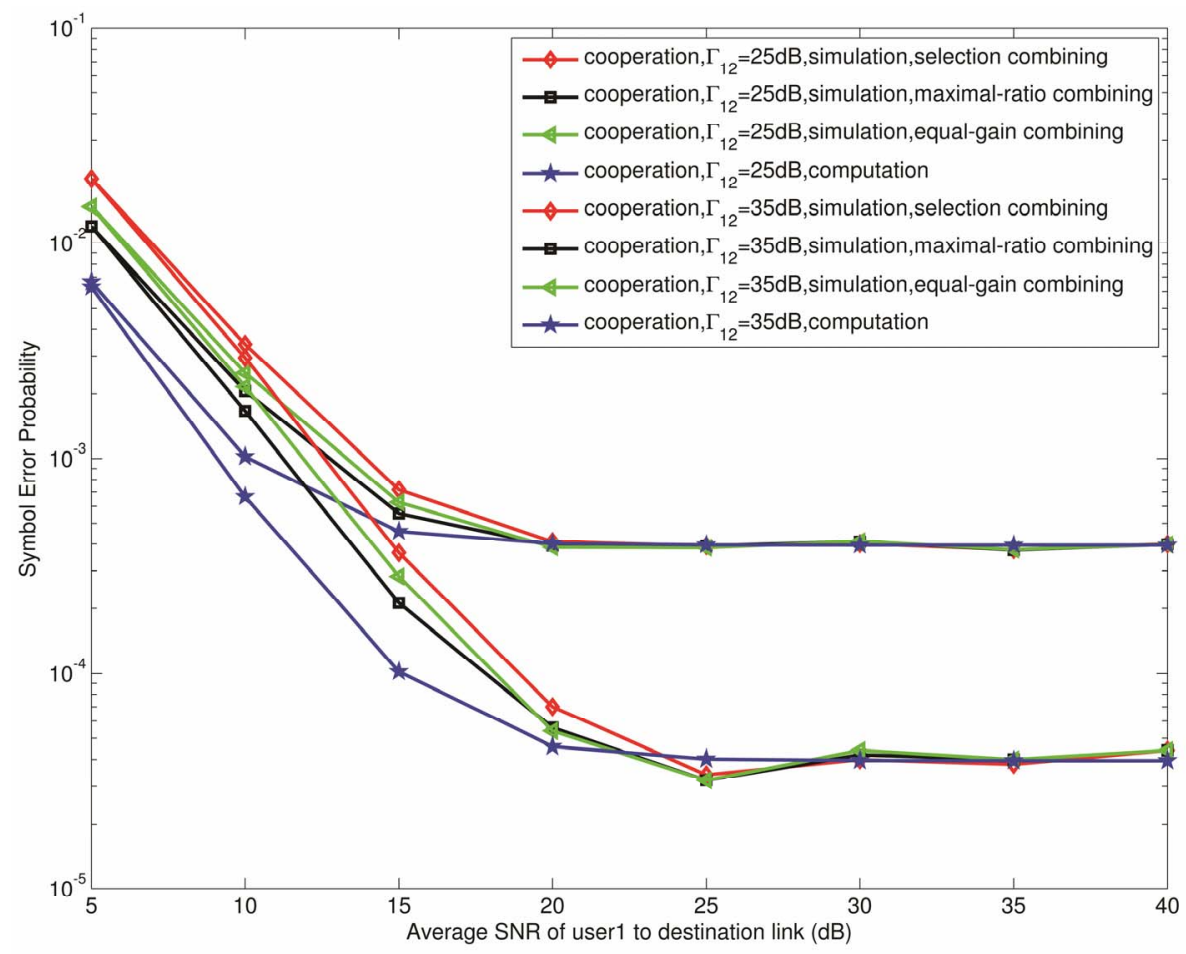

Figure 4. Improved diversity range of MRC for $\Gamma_{12}=25 \mathrm{~dB}, 35 \mathrm{~dB}$.

agree with each other. Further we also presented some choice of SNR values with different combining techniques to obtain low SEP values. Finally we proved that two-user cooperative diversity with MRC implementation was performing better for low SNR values compared to $\mathrm{SC}$ and $\mathrm{EGC}$ techniques.

\section{REFERENCES}

[1] A. Nosratinia, T. E. Hunter and A. Hedayat, "Cooperative Communication in Wireless Networks," IEEE Communications Magazine, Vol. 42, No. 10, 2004, pp. 74-80. doi:10.1109/MCOM.2004.1341264

[2] A. Sendonaris, E. Erkip and B. Aazhang, "User Cooperation Diversity_Part I: System Description," IEEE Tran- sactions on Communications, Vol. 51, No. 11, 2003, pp. 1927-1938. doi:10.1109/TCOMM.2003.818096

[3] J. N. Laneman, D. N. C. Tse and G. W. Wornell, "Cooperative Diversity in Wireless Networks: Efficient Protocols and Outage Behavior," IEEE Transactions on Information Theory, Vol. 50, No. 12, 2004, pp. 3062-3080. doi:10.1109/TIT.2004.838089

[4] M. D. Selvaraj and R. K. Mallik, "Performance Analysis of Two-User Cooperation Diversity," IEEE International Conference on Vehicular Technology, Beijing, 19-23 May 2008, pp. 619-623.

[5] M. O. Hasna and M.-S. Alouini, " End-to-End Performance of Transmission Systems with Relays over RayleighFading Channels," IEEE Transactions on Wireless Communications, Vol. 2, No. 6, 2003, pp. 1126-1131. doi:10.1109/TWC.2003.819030 